\title{
Lymphovascular Invasion 4
}

National Cancer Institute

\section{Source}

National Cancer Institute. Lymphovascular Invasion 4. NCI Thesaurus. Code C147096.

Both lymphatic and small vessel and venous (large vessel) invasion. (AJCC 8th ed.) 